\title{
Realizing 5G- and AI-based doctor-to-doctor remote diagnosis: opportunities, challenges, and prospects
}

\author{
Kenji Karako ${ }^{1}$, Peipei Song ${ }^{2, *}$, Yu Chen ${ }^{1, *}$, Wei Tang ${ }^{3}$ \\ ${ }^{1}$ Department of Human and Engineered Environmental Studies, Graduate School of Frontier Sciences, The University of Tokyo, Chiba, Japan; \\ ${ }^{2}$ Institute for Global Health Policy Research, National Center for Global Health and Medicine, Tokyo, Japan; \\ ${ }^{3}$ International Health Care Center, National Center for Global Health and Medicine, Tokyo, Japan.
}

SUMMARY Fifth Generation (5G) mobile communications technology became available in Japan as of the end of March 2020. The Ministry of Internal Affairs and Communications (MIC) is proceeding with a plan to use $5 \mathrm{G}$ for a doctor-to-doctor remote diagnosis system. This remote diagnosis offers patients the benefit of receiving advanced medical care without having to travel long distances. The provision of a remote diagnosis will provide elderly patients in rural areas with an earlier diagnosis without burdening patients in Japan where the aging population and the uneven distribution of doctors are increasing. However, the system will increase the burden on specialists by expanding the doctor's catchment area. As a solution to that problem, deep learning-based artificial intelligence (AI) is expected to reduce the burden on doctors. In order to realize 5G- and AI-based real-time diagnostic support, diagnostic imaging using $\mathrm{AI}$ and an $\mathrm{AI}$ model that provides instructions are required. This is because ultrasonography and endoscopy, which can be used for remote diagnosis, do not acquire data on fixed areas like a CT or MRI scan. The AI model needs to instruct the doctor at the patient's home in order to collect appropriate information in accordance with the patient's symptoms and status. In order to build an interactive AI model, the interactions between doctors who are making a remote diagnosis should be recorded as training data and a $5 \mathrm{G}$-based remote diagnosis system should be created. A remote diagnostic support system incorporating $5 \mathrm{G}$ and interactive diagnostic imaging incorporating AI will result in a system that places less of a burden on patients and doctors.

Keywords remote diagnosis, 5G mobile communications, artificial intelligence, ultrasonography, endoscopy, Japan

\section{Doctor-to-doctor remote diagnosis}

A remote diagnosis means that a doctor provides medical care to a distant patient via the Internet. This is expected to remedy regional differences in the quality of medical care and to provide high-level medical care to patients on isolated islands and in remote areas. In addition to conventional remote diagnostic imaging and remote pathological diagnosis, new types of remote care such as remote surgical support and remote intensive care, which can be called "urban medicine delivered remotely", have emerged in recent years. The demand for online medical care is also increasing because of COVID-19. In Japan, online medical care was clearly defined by the revision of "Guidelines for Proper Implementation of Online Medical Care" in July 2019 (1). In addition, medical fees were revised this year (2), and the case where a doctor in a remote location provides medical treatment using an information communication device under some conditions will be counted as a medical fee.

There are two major formats for telemedicine, "Doctor-to-Doctor-to-Patient" and "Doctor-to-Patient with Doctor". The first doctor is a specialist who has richer knowledge and clinical experience but works at the hospital located away from the attending physician and the patient. The second doctor is the patient's attending physician who can actually contact the patient. The major difference between the two methods is the way in which a specialist is involved. In the "Doctorto-Doctor-to-Patient" format, the attending physician and the specialist exchange information based on information obtained from the patient by the attending physician, and the attending physician conducts a formal examination and makes a diagnosis based on the advice of the specialist. In contrast, in the "Doctorto-Patient with Doctor" format, the specialist examines and diagnoses the patient with support and information shared by the attending physician near the patient. The 
attending physician conducts examinations using testing equipment while receiving specialized knowledge and technical guidance from the specialist.

\section{The 5G era: Opportunities for doctor-to-doctor remote diagnosis}

In Japan, 5th Generation (5G) mobile communications technology became available from Japan's telecommunications carriers, DoCoMo, au, and SoftBank as of the end of March 2020 (3-5). In conjunction with this, the Ministry of Internal Affairs and Communications (MIC) is proceeding with a plan to use $5 \mathrm{G}$ for telemedicine (6). $5 \mathrm{G}$ is a new generation of mobile communications and has a higher communication speed than the conventional 4th Generation. The main impact of $5 \mathrm{G}$ is the improvement in Internet speed. The maximum downstream speed is about 3.4 Gbps and the maximum upstream speed is about 182 Mbps (3), enabling real-time transmission of $4 \mathrm{~K}$ and high-quality video. This facilitates Doctorto-Doctor with Patient remote diagnosis, whereby a specialist diagnoses the patient with the attending physician while watching video of the examination performed at the patient's home in real time. This remote diagnostic support gives patients the benefit of receiving advanced medical care without having to travel long distances. The provision of remote diagnostic support will provide elderly patients in rural areas with an earlier diagnosis without burdening patients in Japan where the aging population and the uneven distribution of doctors are increasing.

$5 \mathrm{G}$ is expected to allow real-time remote diagnosis via high-quality video. However, there is no great advantage in using it in hospitals that can use optical communications because $5 \mathrm{G}$ is for mobile communications. $5 \mathrm{G}$ is effective when video or data needs to be communicated in real time from a place other than a hospital. Situations involving $5 \mathrm{G}$ and its advantages and requirements are summarized in Table 1. The mobility of equipment for each examination used in remote diagnosis is summarized in Table 2. Table 1 shows communication between hospitals does not require $5 \mathrm{G}$ if there are existing wired connections. In contrast, $5 \mathrm{G}$ is highly useful when making a remote diagnosis at a patient's home, and especially when making a diagnosis based on imaging such as ultrasonography or endoscopy. Aging of the population is progressing and the number of doctors is unevenly distributed in Japan. Here, remote diagnostic support will make it easier for elderly people living in areas far away from hospitals with specialists to receive a diagnosis. In addition, in the new era of COVID-19, the elderly - who are highly susceptible - can benefit from remote diagnostic support without crowding into hospitals. Preparations for such a remote diagnosis system are underway. In China, patients with COVID-19 underwent ultrasonography using a 5G-based robot-assisted remote ultrasound system between temporary hospitals and specialists at a hospital $700 \mathrm{~km}$ away (7).

Table 1. Comparison of whether 5th Generation (5G) mobile technology is required to facilitate real-time remote diagnosis between two points

\begin{tabular}{|c|c|c|c|c|}
\hline \multicolumn{2}{|c|}{ Location } & \multirow{2}{*}{ Necessity of 5G } & \multirow{2}{*}{ Advantages of using $5 \mathrm{G}$} & \multirow{2}{*}{ Types of examinations available } \\
\hline Site of diagnosis & Site of examination & & & \\
\hline Hospital & Hospital & $\begin{array}{l}\times \text { It is not necessary if an optical } \\
\text { communications network is available at } \\
\text { both hospitals. }\end{array}$ & None & $\begin{array}{l}\text { Ultrasonography, Endoscopy, } \\
\text { Skin examination, MRI, CT } \\
\text { Scan, X-Ray }\end{array}$ \\
\hline Hospital & Ambulance & $\begin{array}{l}\circ \text { It is necessary because conventional } \\
\text { mobile communications cannot handle } \\
\text { high-quality video in real time. }\end{array}$ & $\begin{array}{l}\text { Real-time remote diagnosis can } \\
\text { be provided by an emergency } \\
\text { specialist }\end{array}$ & $\begin{array}{l}\text { Ultrasonography, Endoscopy, } \\
\text { Skin examination }\end{array}$ \\
\hline Hospital & Patient's home & $\begin{array}{l}\Delta \text { It is not necessary if the patient's home } \\
\text { has an optical communications network. }\end{array}$ & $\begin{array}{l}\text { Real-time remote diagnosis } \\
\text { can be provided regardless of } \\
\text { location or region. }\end{array}$ & $\begin{array}{l}\text { Ultrasonography, Endoscopy, } \\
\text { Skin examination }\end{array}$ \\
\hline
\end{tabular}

Table 2. Comparison of the mobility of equipment for type of examination used in remote diagnosis

\begin{tabular}{llll}
\hline Type of examination & $\begin{array}{c}\text { Need to be performed } \\
\text { in real time }\end{array}$ & Type of data & Mobility \\
\hline Ultrasonography & $\circ$ & Video & $\circ$ Mobile examination equipment is being developed. \\
Endoscopy & $\circ$ & Video & $\circ$ Mobile examination equipment is being developed. \\
Skin examination & $\times$ & Multiple images & $\circ$ Examination is possible if using a camera and a PC. \\
MRI & $\times$ & Multiple images & $\Delta$ Examination is possible if there is a vehicle equipped with the equipment. \\
CT Scan & $\times$ & Multiple images & $\Delta$ Examination is possible if there is a vehicle equipped with the equipment. \\
X-Ray & $\times$ & Multiple images & $\Delta$ Examination is possible if there is a vehicle equipped with the equipment. \\
\hline
\end{tabular}




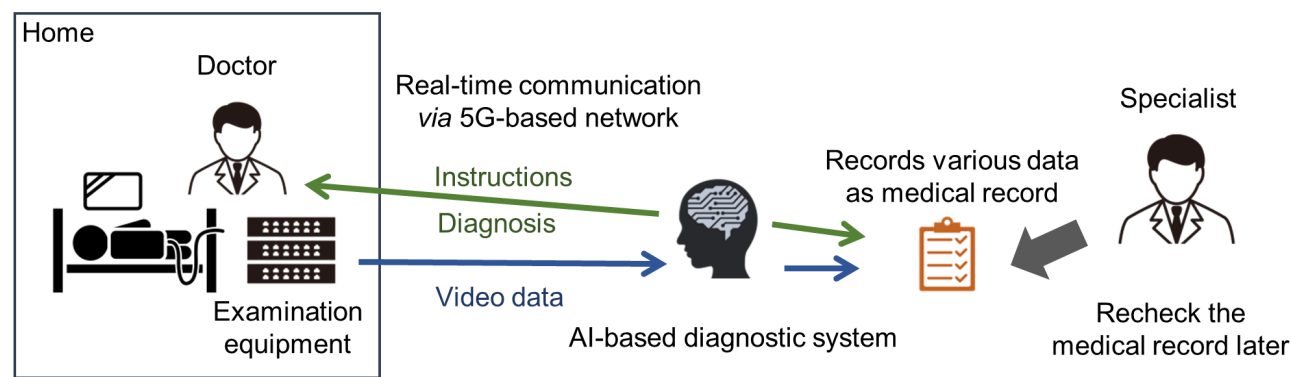

Figure 1. An overview of a 5G-based remote diagnosis system using AI. 5G, 5th Generation; AI, artificial intelligence.

\section{5G era: Challenges for doctor-to-doctor remote diagnosis}

Remote diagnosis is likely to benefit the elderly, especially those living in rural areas, but it is likely to increase the burden on doctors. Remote diagnosis requires at least two doctors: an attending physician who visits the patient's home directly and a specialist who diagnoses the disease and instructs the attending physician. Although the area covered by the attending physician remains the same, remote diagnosis will force a specialist to spend more time diagnosing patients who live in a wider area in addition to his/her current patients.

On the other hands, one of the challenges is securing specialists can realize the remote diagnosis service using $5 \mathrm{G}$ communications technology. By providing remote diagnostic support, mid-sized hospitals are predicted to see patients from an even larger area. However, the number of specialists is limited, and the number of patients per specialist will increase. In other words, remote diagnostic support will decrease the burden on elderly in rural areas and increase the burden on doctors. Faced with this challenge, deep learning is expected to be widely used as a technology to reduce the burden on doctors. In recent years, deep learning has performed well in image recognition. Many deep learning applications involving CT (8) or MRI (9) scans have been proposed, resulting in highly accurate image interpretation. In addition, deep learning applications using ultrasonography and endoscopy image are also being studied. CT and MRI scans cannot be performed at the patient's home, but ultrasonography and endoscopy can be performed using mobile devices. By combining these mobile devices with $5 \mathrm{G}$ communications technology and deep learning, real-time simple remote diagnostic support can be provided without the actual contact between a specialist and the patient.

\section{Prospects: Realizing 5G- and AI-based doctor-to- doctor remote diagnosis}

Deep learning has been studied for various applications in medicine, though the main one is image recognition
(10-12). This is effective when combined with 5G-based remote diagnosis, which mainly uses diagnostic imaging. Figure 1 shows an overview of a 5G-based remote diagnosis system in which a specialist and an attending physician are supported by deep learning. Deep learning replaces the diagnosis conventionally performed by a specialist, and the specialist performs a final check after the remote diagnosis is made. This reduces the burden on specialists. Because the obtained images can be saved, the images that deep learning has used to make a diagnosis can be checked again. Not only can the burden on the doctor be reduced, but the patient's waiting time can also be greatly shortened through the convenient application of remote diagnosis.

However, it is difficult to realize $5 \mathrm{G}$-based artificial intelligence (AI) remote diagnosis support by combining the current technologies. There are two reasons. The first is the types of images that are mainly obtained using ultrasonography or endoscopy in remote diagnosis, but well-calibrated deep learning applications in medicine involve CT and MRI. Ultrasonography and endoscopy do not acquire data on fixed areas like a CT or MRI scan, the angle and position of imaging change depending on the state of the operator and the patient. This means that more varied images are expected to be obtained, and more training data are required to construct a highly accurate AI model. The second reason is that the specialist not only makes a diagnosis based on images but also gives additional instructions to the operator depending on examination results and the patient's condition obtained in remote diagnosis. Similarly, AI needs to make a diagnosis based on the received video and to give instructions to the operator who lacks specialized knowledge in 5G-based AI remote diagnosis. Little research has been conducted in this area, while diagnostic imaging is currently being studied in multiple areas of medicine. In order to provide a remote diagnosis incorporating $\mathrm{AI}$ in the future, instructions given to the operator depending on the patient's condition should be recorded and collected as learning data.

Finally, the potential of introducing AI into a remote diagnosis is expected to be used not only for diagnosis but also for matching specialists and patients. As $5 \mathrm{G}$-based remote diagnosis removes the distance between 
patients and specialists, patients will have more choices. At that time, AI could also be used to find the specialist suitable for the patient's symptoms and condition.

In conclusion, $5 \mathrm{G}$ communications technology allows a specialist to make a diagnosis remotely in real time using images from examinations such as ultrasonography and endoscopy. This will reduce the burden of travel for patients and make it easier for people in the suburbs and the elderly to receive an expert diagnosis. The obstacles posted by distance have disappeared, but specialists must oversee more patients, and the burden on specialists may increase. Remote diagnostic support using AI is expected to be a solution to this problem. Real-time diagnosis using AI enables an examination in accordance with the patient's schedule. In addition, video can be saved so a specialist can check the examination results later.

Funding: None.

Conflict of Interest: The authors have no conflict of interest to disclose.

\section{References}

1. Ministry of Health, Labor, and Welfare. Guidelines for Proper Implementation of Online Medical Care. https:// www.mhlw.go.jp/content/000534254.pdf (accessed September 28, 2020) (in Japanese)

2. Ministry of Health, Labor, and Welfare. An overview of revised medical fees. https://www.mhlw.go.jp/ content/12400000/000616842.pdf (accessed September 28, 2020) (in Japanese)

3. NTT docomo. Press Release: "5G" service launched. https:// www.nttdocomo.co.jp/info/news_release/2020/03/18_00. html (accessed September 30, 2020) (in Japanese)

4. KDDI. "UNLIMITED WORLD au 5G" starts. $5 \mathrm{G}$ mobile communication service "au $5 \mathrm{G}$ " starts on March 26. https://news.kddi.com/kddi/corporate/ newsrelease/2020/03/23/4326.html (accessed September 30, 2020) (in Japanese)

5. SoftBank. Press Release: The commercial service "SoftBank 5G" starts on March 27. https://www.softbank. jp/corp/news/press/sbkk/2020/20200305_05/ (accessed September 30, 2020) (in Japanese)

6. Ministry of Internal Affairs and Communications. Report on a survey of the status of implementation of doctor-todoctor remote medicine https://www.soumu.go.jp/main content/000699422.pdf (accessed September 28, 2020) (in Japanese)

7. Ye R, Zhou X, Shao F, Xiong L, Hong J, Huang H, Tong W, Wang J, Chen S, Cui A, Peng C, Zhao Y, Chen L. Feasibility of a 5G-based robot-assisted remote ultrasound system for cardiopulmonary assessment of patients with COVID-19. Chest. 2020; S0012-3692(20)31870-5.

8. Domingues I, Pereira G, Martins P, Duarte H, Santos J, Abreu P. Using deep learning techniques in medical imaging: A systematic review of applications on CT and PET. Artif Intell Rev. 2019; 53:4093-4160.

9. Mazurowski MA, Buda M, Saha A, Bashir MR. Deep learning in radiology: An overview of the concepts and a survey of the state of the art with focus on MRI. J Magn Reson Imaging. 2019; 49:939-954.

10. Milletari F. Navab N, Ahmadi S. V-Net: Fully convolutional neural networks for volumetric medical image segmentation. 2016; arXiv:1606.04797.

11. Zhang J, Xie Y, Wu Q, Xia Y. Medical image classification using synergic deep learning. Med Image Anal. 2019; 54:10-19.

12. Ker J, Wang L, Rao J, Lim T. Deep learning applications in medical image analysis. IEEE Access. 2018; 6:93759389.

Received October 2, 2020; Revised October 18, 2020; Accepted October 21, 2020.

*Address correspondence to:

Peipei Song, Institute for Global Health Policy Research, National Center for Global Health and Medicine, Tokyo, Japan. E-mail: ppsong-tky@umin.ac.jp

Yu Chen, Department of Human and Engineered Environmental Studies, Graduate School of Frontier Sciences, The University of Tokyo, Chiba, Japan.

E-mail: chen@edu.k.u-tokyo.ac.jp

Released online in J-STAGE as advance publication October 25, 2020. 